EGU21-12295

https://doi.org/10.5194/egusphere-egu21-12295

EGU General Assembly 2021

(c) Author(s) 2021. This work is distributed under

the Creative Commons Attribution 4.0 License.

\title{
Multi-Spacecraft Observations of a Unique Type of High-Latitude ICME
}

\author{
Megan Maunder ${ }^{1}$, Claire Foullon ${ }^{1}$, Robert Forsyth ${ }^{2}$, Emma Davies $^{2}$, David Barnes ${ }^{3}$, and Jackie \\ Davies $^{3}$ \\ ${ }^{1}$ CGAFD, Mathematics, CEMPS, University of Exeter, Exeter, UK \\ ${ }^{2}$ The Blackett Laboratory, Imperial College London, London, UK \\ ${ }^{3}$ STFC, Rutherford Appleton Laboratory, RAL Space, Oxford, UK
}

Coronal Mass Ejections (CMEs) and their interplanetary counterparts (ICMEs) are key drivers of space weather throughout the heliosphere. Observational studies are used to understand their evolution and for developing existing models and theory in space weather forecasting. Motivated by the future exploration of the solar high-latitudes by Solar Orbiter and complimented by Parker Solar Probe, we aim to contribute to the understanding of high-latitude CMEs as they develop into ICMEs. We examine a high-latitude CME and its subsequent ICME using data from STEREO, Ulysses, and near-Earth spacecraft. We apply a triangulation method to the remote-sensing images from the twin STEREO spacecraft and conduct a multi-spacecraft analysis using the in-situ Ulysses, STEREO, and near-Earth spacecraft data. The Ulysses observations, supported by the other spacecraft, provides a clear picture of the ICME geometry and structure: a shock, followed by a sheath region, and a magnetic flux rope followed by a high-speed stream. This ICME differs from the known 'over-expanding' types observed in the high-latitudes by the Ulysses mission, in that it straddles a region between the slow and fast solar winds which in itself drives a shock. 